\title{
Enhanced penetration into 3D cell culture using two and three layered gold nanoparticles
}

This article was published in the following Dove Press journal:

International Journal of Nanomedicine

23 September 2013

Number of times this article has been viewed

\author{
Christopher G England' \\ Thomas Priest ${ }^{2}$ \\ Guandong Zhang ${ }^{2}$ \\ Xinghua Sun ${ }^{2}$ \\ Dhruvinkumar N Patel $^{2}$ \\ Lacey R McNally,4 \\ Victor van Berkel ${ }^{4,5}$ \\ André M Gobin ${ }^{2}$ \\ Hermann B Frieboes ${ }^{1,2,4}$ \\ 'Department of Pharmacology \\ and Toxicology, ${ }^{2}$ Department of \\ Bioengineering, ${ }^{3}$ Department of \\ Medicine, ${ }^{4}$ James Graham Brown \\ Cancer Center, ${ }^{5}$ Department of \\ Surgery, University of Louisville, \\ $\mathrm{KY}, \mathrm{USA}$
}

Correspondence: Hermann B Frieboes Department of Bioengineering, Lutz Hall 419, University of Louisville, Louisville, KY 40208, USA

Tel +l 5028523302

$\mathrm{Fax}+\mathrm{I} 5028526802$

Email hbfrie0I@louisville.edu

\begin{abstract}
Nano-scale particles sized 10-400 nm administered systemically preferentially extravasate from tumor vasculature due to the enhanced permeability and retention effect. Therapeutic success remains elusive, however, because of inhomogeneous particle distribution within tumor tissue. Insufficient tumor vascularization limits particle transport and also results in avascular hypoxic regions with non-proliferating cells, which can regenerate tissue after nanoparticle-delivered cytotoxicity or thermal ablation. Nanoparticle surface modifications provide for increasing tumor targeting and uptake while decreasing immunogenicity and toxicity. Herein, we created novel two layer gold-nanoshell particles coated with alkanethiol and phosphatidylcholine, and three layer nanoshells additionally coated with high-densitylipoprotein. We hypothesize that these particles have enhanced penetration into 3-dimensional cell cultures modeling avascular tissue when compared to standard poly(ethylene glycol) (PEG)-coated nanoshells. Particle uptake and distribution in liver, lung, and pancreatic tumor cell cultures were evaluated using silver-enhancement staining and hyperspectral imaging with dark field microscopy. Two layer nanoshells exhibited significantly higher uptake compared to PEGylated nanoshells. This multilayer formulation may help overcome transport barriers presented by tumor vasculature, and could be further investigated in vivo as a platform for targeted cancer therapies.
\end{abstract}

Keywords: cancer nanotherapy, tumor hypoxia, nanovector transport

\section{Background}

In addition to excessive cellular proliferation, solid tumors typically elicit irregular angiogenesis resulting in structurally abnormal and leaky vascular structures. The passive mechanism termed "enhanced permeability and retention" (EPR) effect ${ }^{1,2}$ takes advantage of this situation by enabling systemically administered nanoparticles sized $10-400 \mathrm{~nm}$ to preferentially extravasate from the vasculature into the interstitial space of solid tumors. Additional factors contributing to nanoparticle pharmacodynamics and cytotoxicity include size, surface charge and morphology.,4 These properties are typically tailored to design systems that exhibit optimal tumor tissue uptake. Drugs delivered via nanoparticles can thus increase treatment effectiveness while reducing systemic toxicity.

In particular, gold nanoparticles have shown promise in cancer treatment. Bulk gold itself is an inert material, though nanoparticles smaller than $5 \mathrm{~nm}$ in diameter have documented cytotoxic properties. ${ }^{5,6}$ Larger particles have demonstrated little cytotoxicity, depending on the surface charge. Cationic particles can be more cytotoxic at lower concentrations compared to anionic nanoparticles, which has been attributed 
to electrostatic interactions of the positively-charged particles with negatively-charged cell membranes. ${ }^{7}$ Surface coatings of poly(ethylene glycol) (PEG) can effectively hide the nanoparticles from the immune system, further lowering systemic toxicity. ${ }^{3,6}$

However, heterogeneity in blood flow due to irregular angiogenesis and vascular remodeling at the tumor site promote tissue hypoxia and thus cell quiescence, presenting a physical barrier to cell-cycle dependent chemotherapeutics delivered by nanocarriers. ${ }^{8-10}$ Inadequate vascularization leads to further impediments that hinder optimal treatment, including insufficient drug dosages due to abnormally long inter-vascular diffusion distances, as well as disturbed convection and diffusion of molecules (such as glucose and oxygen) and nanoparticles in the interstitium, ${ }^{8,11-13}$ as has recently been explored through intravital microscopy and mathematical modeling. ${ }^{11,14}$ Cancerous tissue can contain almost twice the volume of interstitial space compared to normal tissue, ${ }^{8,13}$ an abundance of extracellular matrix proteins along with an increased interstitial pressure may further inhibit nanoparticle delivery and drug diffusion in under-vascularized tumor regions..$^{8,13,15}$ These physical barriers, coupled with intrinsic resistance mechanisms at the cell-scale, often cause cancer drug therapies to fail. Recently, engineering and physics-based approaches are being applied to help tackle these challenges in cancer treatment. ${ }^{16}$

Estimates of nanoparticle distribution in 3D tissue have been previously obtained in collagen gels ${ }^{17}$ as well as in $3 \mathrm{D}$ cell culture. ${ }^{18,19}$ Gold nanoparticle accumulation was shown to be size dependent, suggesting that particles $\sim 20 \mathrm{~nm}$ or smaller had superior penetration abilities compared to larger particles. ${ }^{18}$ For example, particles $<10 \mathrm{~nm}$ were able to better penetrate breast cancer tumor spheroids representing avascular tumor tissue than larger particles, which has also been confirmed in vivo. ${ }^{19}$ It has been shown that while larger polymeric micelles distribute within hypervascularized tumors, only sub-100 nm particles were able to meaningfully penetrate hypoxic tumors. ${ }^{20}$ However, smaller particles $(<0 \mathrm{~nm})$ are not as useful for drug delivery compared to larger nanoparticles, since they are typically unaffected by the EPR effect and are thus less targeted. ${ }^{19}$ Previous studies have shown cellular uptake of both smaller citrate gold nanoparticles and larger silica gold nanoshells, with enhanced cellular uptake of the smaller particles. ${ }^{21-23}$

In this study we examine the uptake of functionalized silica gold nanoshells into a 3-dimensional (3D) cell culture (spheroid) model as a first step to represent avascular liver, lung, and pancreatic tumor tissue. In addition to the nanoshells, citrate gold nanoparticles were investigated for comparison. Cells aggregate in $3 \mathrm{D}$ culture to create avascular nodules with the production of extracellular matrix components and the establishment of diffusion gradients of oxygen, glucose, and nanoparticles. ${ }^{9}$ Experiments with $3 \mathrm{D}$ cell cultures have previously helped to analyze the effect of under-vascularization on particle and drug delivery. ${ }^{18,24}$ Here, particle surfaces were functionalized by a process consisting of either two or three layers to optimize uptake into tumor tissue. Two layer particles were synthesized with an inner layer of alkanethiol and an outer layer of phosphatidylcholine (PC), a type of phospholipid that is a major component of biological membranes. ${ }^{25}$ In order to further optimize tumor uptake, active targeting was promoted by adding a layer of high density lipoprotein (HDL), since the HDL receptor is mainly expressed by liver and cancer cells, thus creating a three layered particle. Size, zeta potential, and morphology were optimized, and the uptake and distribution were compared to the performance of PEGylated nanoparticles.

\section{Methods}

\section{Synthesis of citrate gold nanoparticles}

Particles were synthesized using the method in which gold chloroauric acid is reduced by trisodium citrate. ${ }^{26}$ In this process, 2.2-2.4 mL 1\% weight/volume (wt/v) citrate (Fisher Scientific, Waltham, MA, USA) and $200 \mathrm{~mL} \mathrm{0.01 \%} \mathrm{wt/v}$ $\mathrm{HAuCl}_{4}$ (Alfa Aesar, Ward Hill, MA, USA) are mixed and heated to boiling, which promotes the reaction of sodium citrate to citric acid. Once the reaction is completed, the solution is concentrated using a rotovapor (Buchi Rotovapor System, BÜCHI Labortechnik AG, Flawil, Switzerland) to $\sim 20 \mathrm{~mL}$ before the addition of layering to the particles.

\section{Synthesis of silica gold nanoshells}

Particles have an inner core composed of silica with an outer coating of gold. Synthesis follows the Stöber method, ${ }^{27-29}$ which consists of four stages: production of small gold seeds, fabrication of monodispersed silica cores, attachment of gold to the seeds, and gold shell growth. The gold colloid solution is created using the recipe from Duff et al. ${ }^{30}$ Growth of the silica cores requires the combination of $7.5 \mathrm{~mL}$ tetraethylorthosilicate (Sigma Aldrich, St Louis, MO, USA), $225 \mathrm{~mL}$ absolute ethanol (Decon Labs, King of Prussia, PA, USA), and $12.5-13.5 \mathrm{~mL}$ ammonia (Sigma Aldrich). ${ }^{31}$ Ammonia is adjusted to achieve silica core sizes $110 \pm 5 \mathrm{~nm}$. After removal of the paraffin cover and evaporation of the ammonia, the cores are coated with 3\%-4\% aminopropyltriethoxysilane (APTES; Sigma Aldrich). Once the seeds are washed, the 
$10 \%$ gold solution is added. After reaction time, the seeds are washed and redispered in DI water. The seeds are diluted to $0.3-0.5$ optical density (OD) at $530 \mathrm{~nm}$ (Varian Cary 50 Bio UV-Visible Spectrometer, McKinley Scientific, Sparta, NJ, USA). A sweep of the seeds is performed to optimize the chemical ratio between them, $\mathrm{K}_{2} \mathrm{CO}_{3}-\mathrm{HAuCl}_{4}$, and formaldehyde (Fisher Scientific).

\section{Particle functionalization}

The first layer applied to the citrate gold nano particles was Hexadecanethiol (Sigma Aldrich) dissolved in ethanol. ${ }^{32}$ While stirring, $20 \mathrm{~mL}$ pure ethanol (Decon Labs) was placed in a beaker with $60 \mu \mathrm{L} 1$-Hexadecanethiol being added secondly. The nanoparticles were added to the sample slowly over the next few minutes. The sample was first agitated for 20 minutes, then placed for 12 hours on an orbital rocker (Boekel Scientific, Feasterville, PA, USA). The sample was spun down and the pellet was washed twice and resuspended in chloroform (Sigma Aldrich). The second functionalization was the addition of the phosphatidylcholine (PC). The stock solution was made by diluting phosphatidylcholine in chloroform, and $100 \mu \mathrm{L}$ were added to the particles after the thiol layer and allowed to set overnight on an orbital rocker. The solutions were transferred to glass tubes and the chloroform evaporated at ambient temperature. This process completed the two layered particles, referred to as citrate gold/thiol/phosphatidylcholine $\left(\mathrm{C}_{\mathrm{AU}} / \mathrm{TL} / \mathrm{PC}\right)$ and nanoshell/ thiol/phosphatidylcholine (NS/TL/PC). The three layered particles, referred to as $\mathrm{C}_{\mathrm{AU}} / \mathrm{TL} / \mathrm{PC} / \mathrm{LP}$ and NS/TL/PC/LP, were created by optimizing the ratio HDL to paticle optical density, and allowed to react overnight.

PEGylation is considered a common form of surface modification for delivering nanoparticles into cancerous tissue, thus we created PEGylated nanoparticles $\left(\mathrm{C}_{\mathrm{AU}} /\right.$ PEG, citrate gold/PEGylated, and NS/PEG, gold nanoshell/ PEGylated) to compare the efficacy of the synthesized two and three layer nanoparticles. To prepare PEGylated nanoparticles, 2,000 molecular weight (MW) PEG was added at a molar ratio of 2,500:1. The solution was allowed to react overnight on the rocker. Excess PEG was removed by centrifuging the particles at $13,000 \mathrm{x}$ g for 20 minutes. The pellet was resuspended in phosphate buffered saline (PBS). It is well documented that PEGylated particles will stay in circulation longer than non-PEGylated particles. ${ }^{33}$ To create the PEGylated particles, both citrate gold nanoparticles and silica gold nanoshells were incubated with 2,000 MW PEG overnight and then centrifuged to separate the particles.

\section{Particle characterization}

Nanoparticle identity was verified as follows: (1) Maximum absorption wavelengths were obtained using the Varian Cary 50 Bio UV-Visible Spectrometer (McKinley Scientific); (2) size and zeta potential measurements were obtained using the ZetaSizer Nanoseries ZS90 (Malvern Instruments, Worcestshire, UK); (3) shape and size were determined using a Zeiss Supra 35VP (Carl Zeiss, Oberkochen, Germany) scanning electron microscope (SEM); (4) presence of lipids on the particle cores was confirmed using a Fourier transform infrared (FTIR) instrument (Perkin Elmer Spectrum BX; Perkin Elmer, Waltham, MA, USA) and through visual analysis using the SEM.

\section{Cell culture and particle experiments}

Human lung adenocarcinoma (A-549) and liver hepatocellular carcinoma (HEPG2) cells were maintained in Dulbecco's Modified Eagle's Medium (Sigma Aldrich) with 10\% fetal bovine serum and $1 \%$ penicillin-streptomycin-glutamine solution in a humidified atmosphere of $5 \% \mathrm{CO}_{2}$ at $37^{\circ} \mathrm{C}$. Pancreatic adenocarcinoma (S2VP10) cells were maintained with RPMI 1640 medium (Cellgro, Corning Inc.) in similar conditions. All cells were grown to $80 \%$ confluence before $3 \mathrm{D}$ cell culture formation. Using 24 well ultra-low attachment plates (Corning Inc., Corning, NY, USA), 100,000 cells were placed in each well and lightly shaken for $\sim 15$ minutes. Tumor spheroid formation occurred by self-aggregation after 7-14 days of incubation. Spheroids typically measured between 0.5 and $2 \mathrm{~mm}$ in diameter, though the S2VP10 cells produced smaller, grape-like structures. Spheroids were incubated with $40 \mu \mathrm{L}$ particles at 25 OD for 6 hours before being washed with PBS.

\section{D cell culture cryosectioning and creation of tissue slides}

$3 \mathrm{D}$ cell cultures were placed in cryomolds made of tissue freezing medium. Samples were allowed to set for 2 hours, and then sectioned at $5 \mu \mathrm{m}$ using a Leica CM1860 Cryostat (Leica Microsystems, Solms, Germany). Sectioned spheroids were then fixed onto Superfrost Plus microscope slides (Fisher Scientific). Successful fixation was determined via bright-field microscopy. Excess media was removed by soaking with 30\% neutral buffered formalin for 1 minute. Slides were washed in deionized water prior to analysis. All experiments were done in triplicate.

\section{Particle detection using Silver Enhancement Stain}

Slides were placed in cold acetone (Fisher Scientific) for 30 seconds, then allowed to dry for 1 minute. Slides were 
placed in 10\% formalin buffer (Sigma Aldrich) for 3 minutes, then washed two times with deionized water and allowed to dry for 5 minutes. The silver enhancement solution was prepared by mixing $1 \mathrm{~mL} /$ slide Silver Enhancement Stain A along with $1 \mathrm{~mL} /$ slide Silver Enhancement Stain B (Sigma Aldrich). The two solutions were mixed in a $50 \mathrm{~mL}$ tube and vortexed for $10-15$ seconds. The combined solution $(2 \mathrm{~mL})$ was added to each slide and allowed to react for 6 minutes. After the reaction time, the solution was washed two times using deoinized water. Slides were analyzed using NIS Elements AR and an Accuscope 3032 inverted light microscope. Region of interest (ROI) intensity measurements were recorded for both stained and unstained samples.

\section{Particle detection using hyperspectral imaging}

The CytoViva Hyperspectral Imaging System (CytoViva Inc., Auburn, AL, USA) was used to locate nanoparticles within sections of $3 \mathrm{D}$ cell cultures. A Dage camera with the dark field microscope was used to obtain images highlighting the nanoparticles. Particle presence was confirmed using hyperspectral imaging, with data analysis performed using ENVI 4.8 and ImageJ (Exelis Visual Information Solutions, Boulder, CO, USA). Using the software, linear (0-255) enhancement was applied and the z-profile library was used to detect single nanoparticles within $3 \mathrm{D}$ cell cultures. ROIs were determined and particles within these regions were counted to determine concentration in various tumor areas. The number of particles decreased from the periphery towards the center; accordingly, measurements from 10 to 50 $\mu \mathrm{m}$ from the tumor periphery were taken to determine spatial distribution of particles into the tumor spheroids.

\section{Statistical analysis}

Analyses used the two tailed Student's $t$-test with significant values being less than 0.05 . Statistically significant results are illustrated with an asterisk in Figure 5.

\section{Results}

\section{Nanoparticle characterization}

Silica gold nanoshell and citrate gold particles were layered with either two or three layers (Figure 1) to promote enhanced penetration into 3D cell cultures of various cancer cell lines, while comparing their penetration and diffusion capabilities to previously designed PEGylated nanoparticles. Nanoparticles were characterized with ultraviolet (UV)-visible (UV-Vis) absorbance spectroscopy, zeta potential analysis, dynamic light scattering (DLS), scanning electron microscopy, and FTIR to ensure proper particle formation and modification.
Optical and charge measurements (Figure 2) indicated that the surface modifications were successful. Prepared citrate gold nanoparticles (Figure 2A) had a peak absorbance between 533-541 nm (Table 1), which is consistent with previous work. ${ }^{34}$ Silica-gold nanoshells (Figure $2 \mathrm{~B}$ ) exhibited peak absorbance between $820-860 \mathrm{~nm}$ (Table 1), which is typical for nanoshells having $110 \mathrm{~nm}$ diameter silica cores and a $\sim 15 \mathrm{~nm}$ gold coating. ${ }^{35}$ The spectra displayed minute amounts of noise at higher wavelengths, which is expected for silica gold nanoshells. ${ }^{36}$ Nanoshells demonstrated ideal optical properties for near-infrared (NIR) application, ${ }^{37,38}$ though such experiments were not included in the scope of this work.

In order to verify particle surface modifications, the zeta potential was measured (Figure 2C and 2D). Modification with $\mathrm{PC}$ and thiol resulted in more highly negatively charged particles compared to PEGylated particles. Conversely, highdensity lipoprotein caused the zeta potential to become more positively charged when compared to PEGylated particles, but still yields slightly negatively charged particles. PEGylated citrate gold nanoparticles exhibited a zeta potential of $-9 \mathrm{mV}$, while the two layer citrate gold nanoparticles had a zeta potential of $-20 \mathrm{mV}$ and the three layer citrate gold nanoparticles had $-2 \mathrm{mV}$. Gold nanoshells generally exhibited higher surface charge than citrate gold nanoparticles, which is consistent with the larger size of the nanoshells. PEGylated nanoshells had a zeta potential of $-18 \mathrm{mV}$, two layer nanoshells measured a zeta potential of $-29 \mathrm{mV}$, and three layer nanoshells had a zeta potential of $-6.8 \mathrm{mV}$. These results are in agreement with previous studies. ${ }^{39-41}$

To ensure the presence of surface modifications, FTIR spectroscopy was performed (Figure 3). PEGylated nanoparticles exhibited $\mathrm{C}-\mathrm{O}-\mathrm{C}$ stretch of the PEG ether $\sim 1050 \mathrm{~cm}^{-1}$ and vibration $\sim 1350 \mathrm{~cm}^{-1}$, along with $-\mathrm{CH}_{2}$ and $-\mathrm{CH}_{3}$ bending vibrations around $\sim 1450 \mathrm{~cm}^{-1}$. This stretch is smaller than previously reported ${ }^{42}$ possibly due to the addition of PEG onto the silica-core nanoshells. Two layer nanoparticles with an outer layer of phosphatidylcholine exhibited $\mathrm{PO}_{4}^{3-}$ group vibrations between $\sim 820-1000 \mathrm{~cm}^{-1}, \mathrm{C}-\mathrm{O}-\mathrm{C}$ stretch $\sim 1100 \mathrm{~cm}^{-1}$, with the largest band corresponding to the asymmetric and symmetric $-\mathrm{CH}_{2}\left(2880 \mathrm{~cm}^{-1}\right)$ and $-\mathrm{CH}_{3}\left(2950 \mathrm{~cm}^{-1}\right)$ stretch and vibration, respectively. For HDL coated nanoparticles, the asymmetric and symmetric $-\mathrm{CH}_{2}\left(2880 \mathrm{~cm}^{-1}\right)$ and $-\mathrm{CH}_{3}$ $\left(2950 \mathrm{~cm}^{-1}\right)$ stretch and vibration are still shown along with $\mathrm{C}=\mathrm{O}$ from the lipid ester $\sim 1750 \mathrm{~cm}^{-1}$, amide bonds between $1600-1700 \mathrm{~cm}^{-1}$, and a phospholipid $\mathrm{P}=\mathrm{O}_{2}$ stretch $\sim 1250 \mathrm{~cm}^{-1}$. The two and three layers exhibited overlap in their spectra due to the layering process of the surface modifications. 

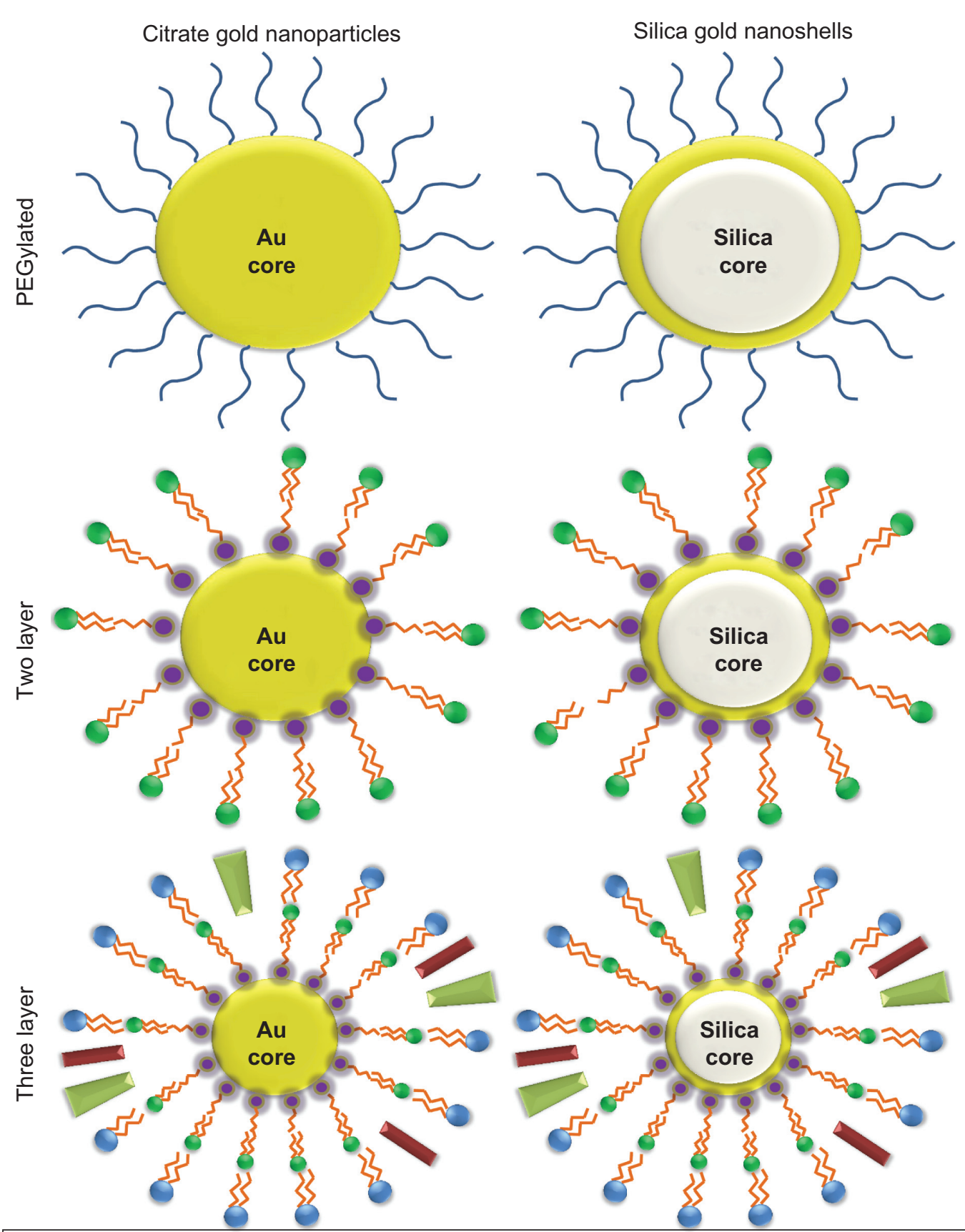

Key: Poly(ethylene)glycol

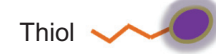

Phosphatidylcholine Au shell

High density lipoprotein HDL (Apo proteins)

HDL (cholesterol)

Figure I Illustration of functionalized silica gold nanoshells and citrate gold nanoparticles.

Notes: Six types of nanoparticles were developed including citrate gold particles and silica gold nanoshells. Particles were modified to form PEGylated, two layer, and three layer particles.

Abbreviations: PEG, poly(ethylene)glycol; Au, gold; HDL, high-density lipoprotein.

SEM was used to determine particle size and morphology (Figure 4). Nanoparticle shape has been demonstrated to influence particle uptake into tissues. ${ }^{6}$ Gold nanoshells, and for the most part citrate gold nanoparticles, were spherical, which is desirable to produce optimal optical properties for potential
NIR treatments. PEGylated gold nanoshells (Figure 4A) had spherical morphology and were $\sim 150-170 \mathrm{~nm}$ in diameter. The influence of hydrodynamic size plays a large role in how nanoparticles will act in vivo. DLS values were determined for each type of particle with varying sizes similar to those depicted 


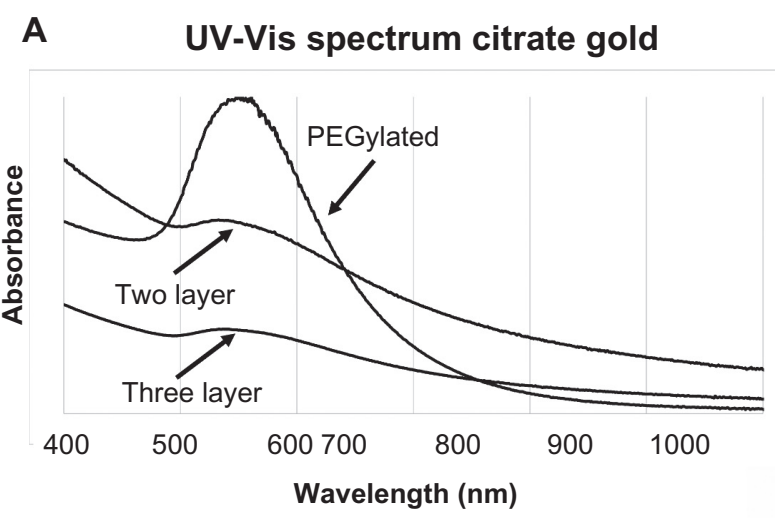

C Zeta potential citrate gold

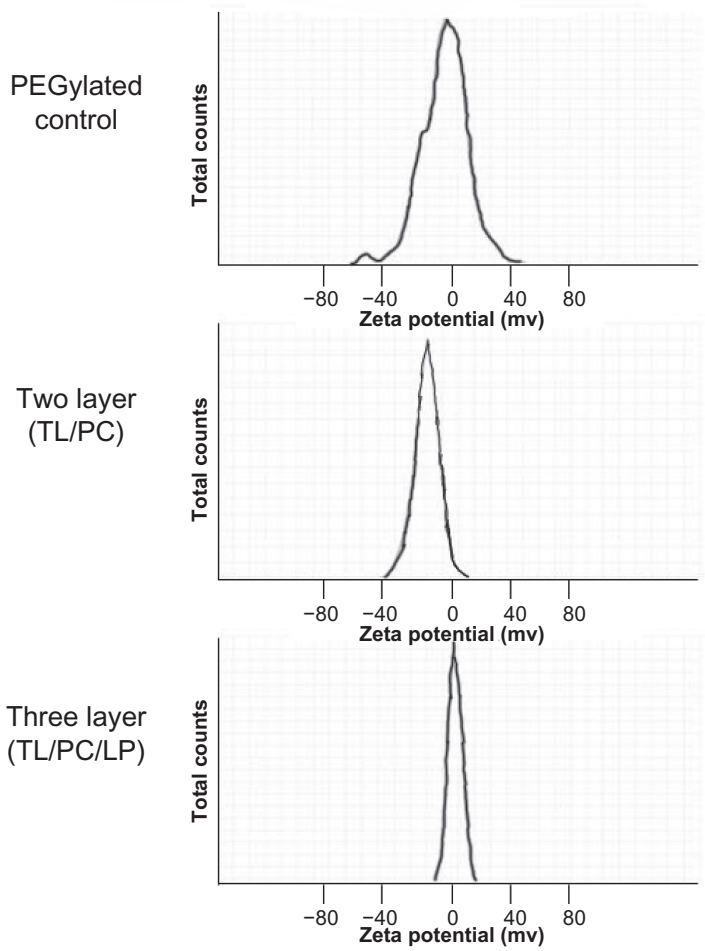

\section{B UV-Vis spectrum silica gold}

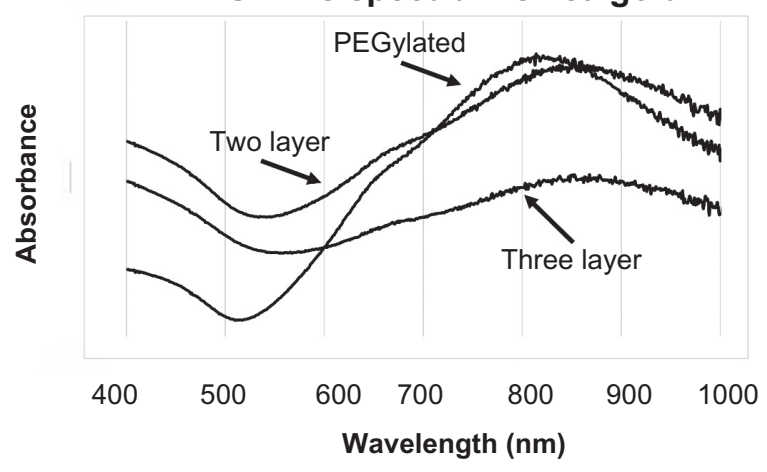

D Zeta potential silica gold
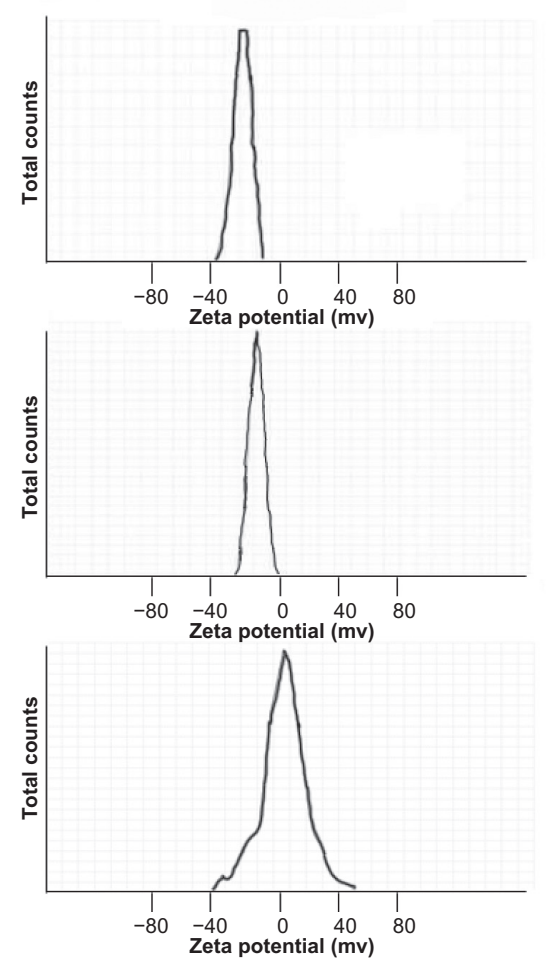

Figure 2 Characterization of citrate gold nanoparticles and silica gold nanoshells by UV-visible spectrometry and zeta potential analysis.

Notes: Wavelengths for each particle are presented with PEGylated particles, two layer, and three layer. (A) Spectrum of citrate gold nanoparticles indicates a maximum absorption ranging from $\sim 533-54 \mathrm{Im}$. (B) Spectrum of silica gold nanoshells suggests a maximum absorption at $\sim 820-860 \mathrm{~nm}$. For verification of surface modifications, zeta potential analysis is shown below the spectra as an indication of surface charge. (C) PEGylated citrate gold nanoparticles had a zeta potential of - $9 \mathrm{mV}$, two layer citrate gold nanoparticles had $-20 \mathrm{mV}$, and three layer citrate gold nanoparticles had $-2 \mathrm{mV}$. (D) A zeta potential of $-18 \mathrm{mV}$ was found for PEGylated silica gold nanoshells, for two layer silica gold nanoshells it was $-29 \mathrm{mV}$, and for three layer nanoshells it was $-6.8 \mathrm{mV}$.

Abbreviations: PEG, poly(ethylene)glycol; UV-Vis, ultraviolet-visible; TL, thiol; LP, lipoprotein. PC, phosphatidylcholine.

by SEM (Table 1). To ensure decreased aggregation before DLS analysis, particles were sonicated and mixed thoroughly.

Citrate gold nanoparticles were much smaller than nanoshells (Figure 4), measuring $\sim 50 \mathrm{~nm}$ in diameter. Aggregation was more prevalent with citrate gold nanoparticles, as evidenced by the PEGylated particles. This aggregation is due to the decreased zeta potential of citrate gold nanoparticles when compared to the gold nanoshells. Addition of hexadecanethiol and $\mathrm{PC}$ is estimated to add $\sim 5-7 \mathrm{~nm}$ to the diameter of the gold nanoparticles due to the size of PC ( 2.5-3.0 nm and -thiol $\sim 2.5 \mathrm{~nm}),{ }^{43,44}$ while HDL is estimated to add $\sim 10 \mathrm{~nm}$ due to the size of this molecule. ${ }^{45}$

\section{Nanoparticle penetration in 3D cell cultures}

In order to determine particle penetration and distribution into 3D cell cultures, ROI measurements were taken at the periphery and center of the spheroid sections using silver 
Table I Determination of nanoparticle size and wavelength

\begin{tabular}{|c|c|c|c|c|c|c|}
\hline & \multicolumn{3}{|c|}{ Citrate gold nanoparticles } & \multicolumn{3}{|c|}{ Silica gold nanoshells } \\
\hline & $\begin{array}{l}\text { SEM } \\
(\mathrm{nm})\end{array}$ & $\begin{array}{l}\text { DLS } \\
(\mathrm{nm})\end{array}$ & $\begin{array}{l}\text { Maximum } \\
\text { wavelength } \\
(\mathrm{nm})\end{array}$ & $\begin{array}{l}\text { SEM } \\
(\mathrm{nm})\end{array}$ & $\begin{array}{l}\text { DLS } \\
(\mathrm{nm})\end{array}$ & $\begin{array}{l}\text { Maximum } \\
\text { wavelength } \\
(\mathrm{nm})\end{array}$ \\
\hline PEGylated (PEG) & $52.6(15.4)$ & $82.81(13.4)$ & 533 & $146.2(9.1)$ & $161.82(12.2)$ & 820 \\
\hline Two layer (TL/PC) & $47.1(12.6)$ & $74.91(13.3)$ & 540 & $144.9(6.5)$ & $155.11(13.9)$ & 855 \\
\hline Three layer (TL/PC/LP) & $62.8(14.9)$ & $85.26(18.7)$ & 541 & I58.| (4.6) & 169.41 (II.8) & 860 \\
\hline
\end{tabular}

Notes: Nanoparticle sizes were determined using SEM and DLS for comparison. Max absorptions from UV-visible spectroscopy are also shown.

Abbreviations: DLS, dynamic light scattering; SEM, scanning electron microscope; UV, ultraviolet; TL, thiol; LP, lipoprotein. PC, phosphatidylcholine; PEG, poly(ethylene)glycol.

enhancement staining (Figure S1). This staining is commonly used for nanoparticle detection. ${ }^{46}$ We also evaluated the samples using hyperspectral imaging with dark field microscopy. ${ }^{47}$ Results (ROI intensity) were measured between unstained and stained samples. Differences between the samples were extracted in order to obtain particle accumulation in the $3 \mathrm{D}$ cell cultures.

For all three cell lines, the two and three layer silica gold nanoshells staining exhibited higher ROI intensity values than the PEGylated counterpart (Figure 5). For the HEPG2 cells, the two layer nanoshells presented ROI intensity values that were statistically the same between periphery and interior, indicating uniform distribution into the tissue. This particle type had an ROI intensity nearly two times that of the three layer and three times that of the PEGylated particles (Figure 5A). The two layer citrate gold particles had a peripheral ROI intensity that was almost two times that of the interior, indicating a steep diffusion gradient into the $3 \mathrm{D}$ cell culture. In contrast, the three layer citrate gold particles exhibited similar intensities between periphery and interior at lower values than the silica gold nanoshells, suggesting uniform yet poorer penetration. The PEGylated particles had higher ROI intensity at the periphery than the interior while showing the lowest particle uptake. It should be noted that ROI intensity differences for the HEPG2 cell line were higher than both the A549 and S2VP10 cell lines, suggesting an increased amount of particles present in the HEPG2 tumors regardless of particle type.

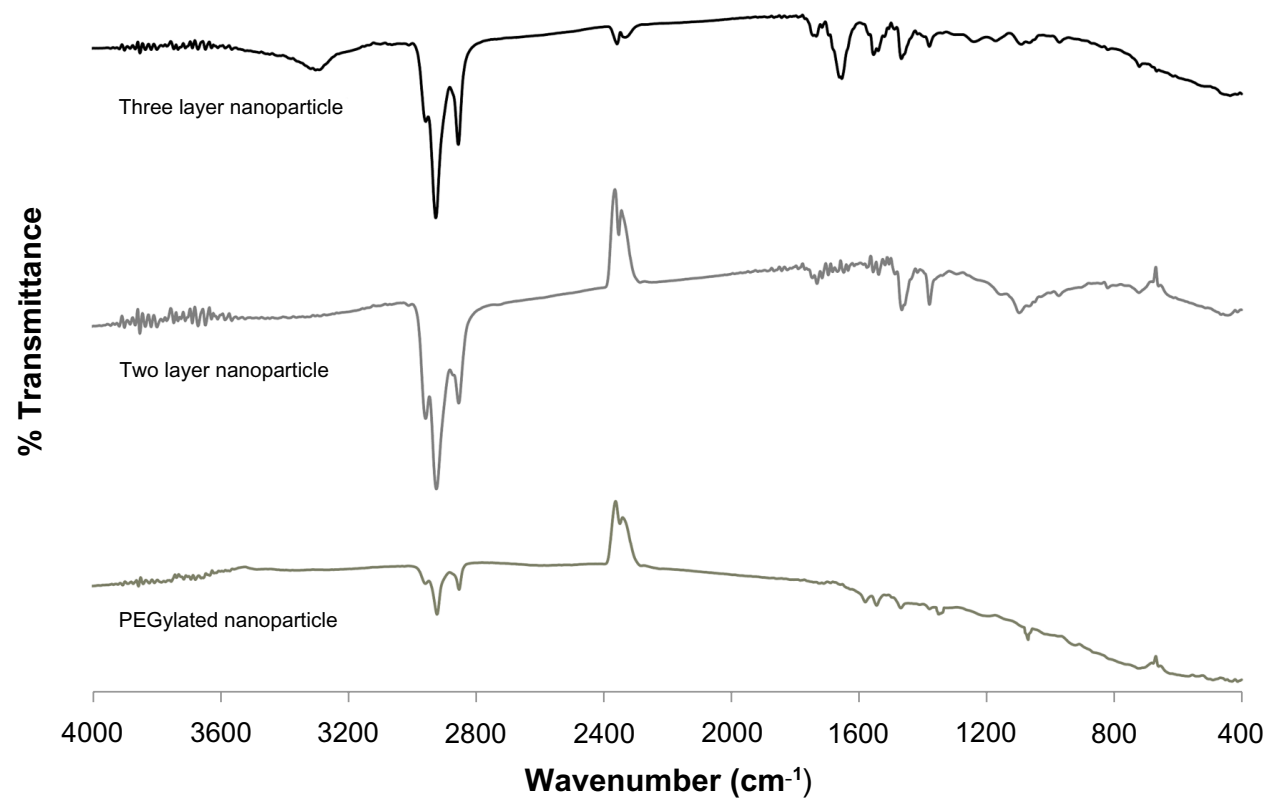

Figure 3 Determination of surface functionalizing using FTIR.

Notes: From bottom to top: PEGylation, two layer containing dodecanethiol and phosphatidylcholine, and three layer containing dodecathiol, phosphatidylcholine and HDL. PEGylated nanoparticles exhibited C-O-C stretch of the PEG ether $\sim 1050 \mathrm{~cm}^{-1}$ and vibration $\sim 1350 \mathrm{~cm}^{-1}$, along with $-\mathrm{CH}_{2}$ and $-\mathrm{CH}_{3}$ bending vibrations around $\sim 1450 \mathrm{~cm}^{-1}$. Two layer nanoparticles with an outer layer of phosphatidylcholine exhibited $\mathrm{PO}_{4}{ }^{3-}$ group vibrations between $\sim 820-1000 \mathrm{~cm}^{-1}, \mathrm{C}-\mathrm{O}-\mathrm{C}$ stretch $\sim 1100 \mathrm{~cm}^{-1}$, with the largest band corresponding to the asymmetric and symmetric $-\mathrm{CH}_{2}\left(2880 \mathrm{~cm}^{-1}\right)$ and $-\mathrm{CH}_{3}\left(2950 \mathrm{~cm}^{-1}\right)$ stretch and vibration, respectively. For $\mathrm{HDL}$ coated nanoparticles, the asymmetric and symmetric $-\mathrm{CH}_{2}\left(2880 \mathrm{~cm}^{-1}\right)$ and $-\mathrm{CH}_{3}\left(2950 \mathrm{~cm}^{-1}\right)$ stretch and vibration are still shown along with $\mathrm{C}=\mathrm{O}$ from the lipid ester $\sim 1750 \mathrm{~cm}{ }^{-1}$, amide bonds between $1600-1700 \mathrm{~cm}^{-1}$, and a phospholipid $\mathrm{P}=\mathrm{O}_{2}$ stretch $\sim 1250 \mathrm{~cm}^{-1}$.

Abbreviations: FTIR, Fourier transform infrared; HDL, high density lipoprotein; PEG, poly(ethylene)glycol. 

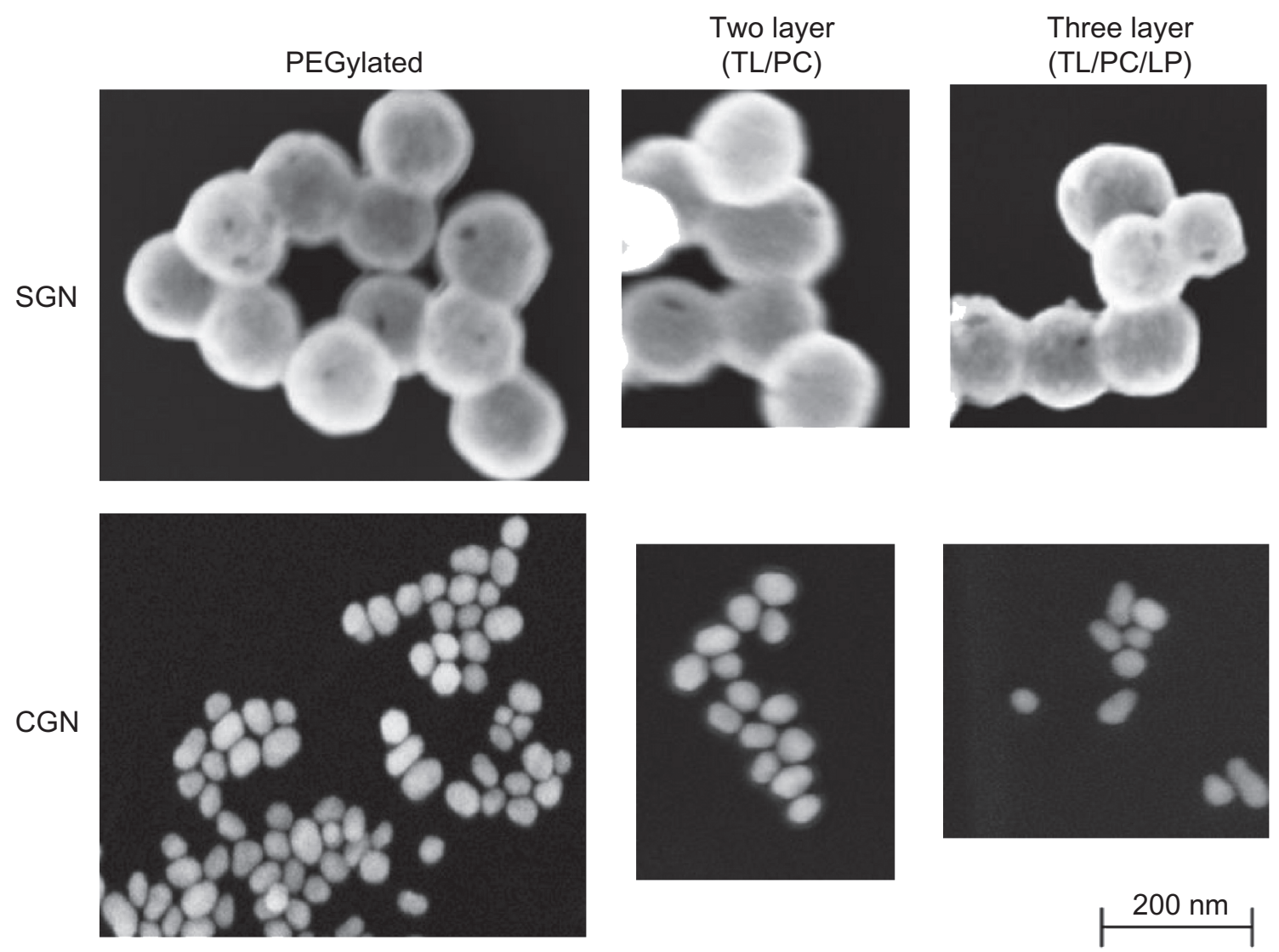

Figure 4 Scanning electron images of functionalized citrate gold nanoparticles and silica gold nanoshells.

Notes: Silica gold nanoshells exhibit a particle size $\sim 150-170 \mathrm{~nm}$ in diameter. Citrate gold nanoparticles with an average diameter of $\sim 50 \mathrm{~nm}$ were much smaller than nanoshells, and appeared to form polydispersed clusters, with the two and three layer citrate gold particles being approximately the same size.

Abbreviations: SGN, Silica Gold Nanoshell; CGN, Citrate Gold Nanoparticle; PEG, poly(ethylene)glycol; TL, thiol; LP, lipoprotein. PC, phosphatidylcholine.

The ROI intensities for the A549 cell line indicated an increased accumulation of two layer compared to PEGylated silica gold nanoshells (Figure 5B). The two layer particle had higher penetration but with more of a differential between periphery and tumor interior compared to the three layer case. The three layer citrate gold particle exhibited higher ROI and more uniform values compared to the other citrate gold cases.

The S2VP10 cell line presented approximately two times the ROI intensity values at the periphery for both two and three layer silica gold nanoshells compared to the PEGylated case (Figure $5 \mathrm{C}$ ), although the three layer had $\sim 50 \%$ higher values than the two layer in the interior. Both two and three layer silica nanoshell and citrate gold particles had significantly higher ROI in the interior than the PEGylated particles, indicating increased particle penetration. The two and three layer citrate gold particles presented similar ROI intensity values between periphery and interior compared to the PEGylated case, suggesting a more uniform particle distribution.

Modified particles within the sectioned tumor spheroids were observable in the images captured via dark-field microscopy. The particles reflected the light, displaying sharp contrast with the surrounding tissue, and could be numerically calculated. A representative image is displayed in Figure S2, and arrows pointing to the small red-orange dots indicate the position of sample particles within the tissue. To ensure that the particles could be distinguished from the tissue, hyperspectral mapping was used to filter out the tissue while leaving nanoparticles. ${ }^{48}$ Spectral libraries, as shown in Figure S3, were used to locate nanoparticles within the 3D cell culture. Individual nanoparticles were identified and accumulation within various spheroid regions was calculated.

Particle uptake and penetration from the periphery of the tumor spheroids into interior regions was analyzed via counts obtained from the hyperspectral dark-field images (Figure 6). In agreement with the silver staining measurements, the two layer silica gold nanoshells in the HEPG2 tumors and the three layer citrate gold nanoparticles in the A549 tumors had the best performance in terms of higher uptake and uniform distribution, while both of these particle types behaved similarly in the S2VP10 tissue with lower uptake and steeper 


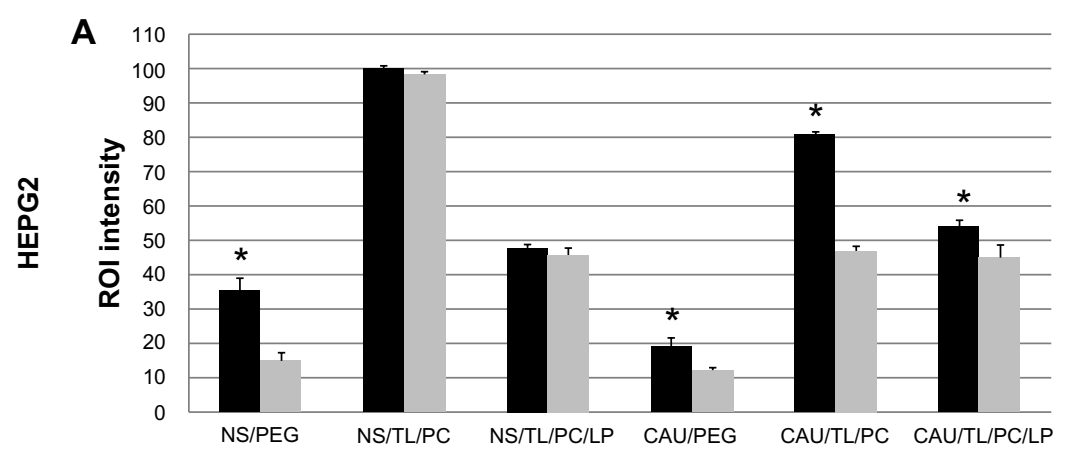

B
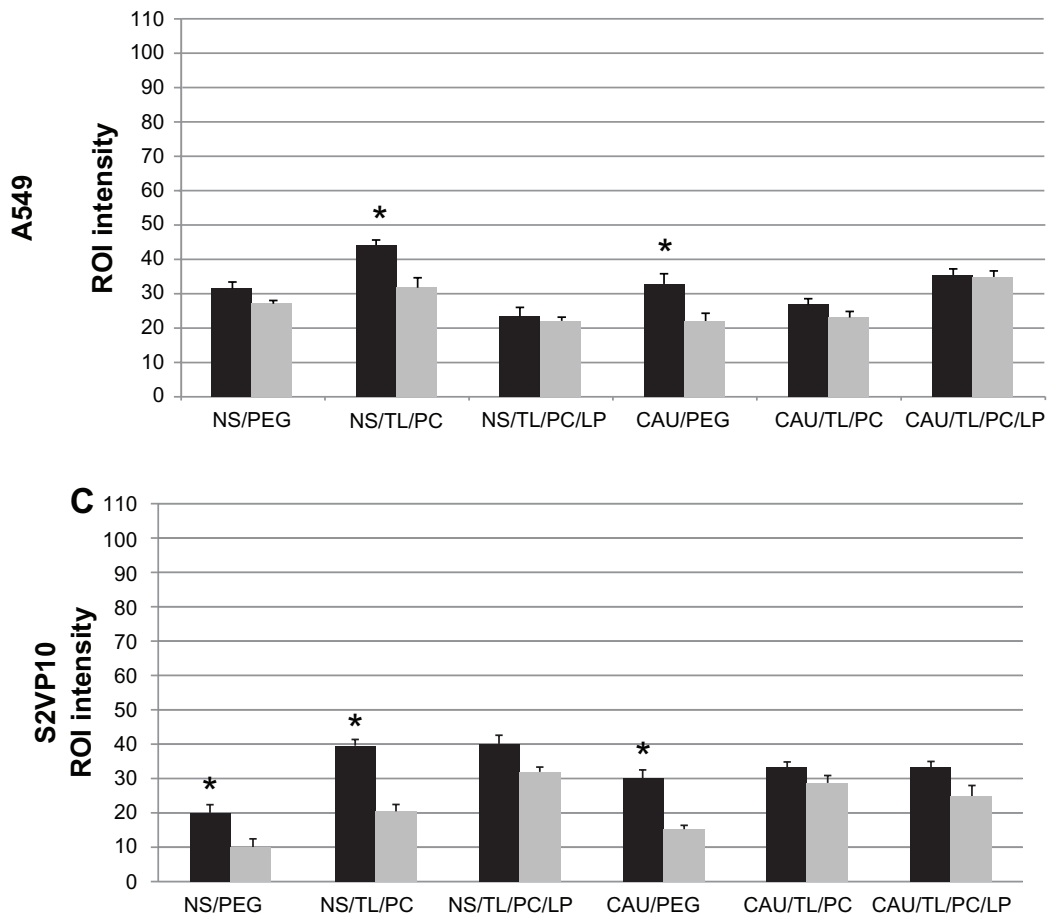

Figure 5 Accumulation of citrate gold nanoparticles and silica gold nanoshells in three dimensional cancer cell cultures as measured by silver enhancement staining. Notes: ROI intensity measurements were taken at the periphery (black bars) and in center regions (grey bars) of HEPG2 (liver), A549 (non-small cell lung cancer), and S2VPIO (pancreatic) 3D cell cultures. Exposure to the various types of silica gold nanoshells and citrate gold nanoparticles generally exhibited equal or increased amounts of particles on the spheroid periphery as compared to the interior. All error bars represent standard deviation from at least $n=3$ measurements; asterisk indicates statistical significance $(p<0.05)$ determined by Student's $t$-test with $\alpha=0.05$.

Abbreviations: 3D, three dimensional; CAU, citrate gold; LP, lipoprotein; NS, nanoshell; PC, phosphatidylcholine; PEG, poly(ethylene)glycol; ROI, region of interest; TL, thiol.

diffusion gradients. The three layer particles showed slightly higher uptake into S2VP10 tissue, which could be due to the morphology of these tumors resembling a cluster of grapes, as well as other cancer-specific interactions with the HDL. Generally, the PEGylated particles exhibited lower uptake and poorer penetration.

Comparing the gold nanoshell results across cancer types, the two layer nanoshells exhibited an average 50\% higher uptake compared to the three layer nanoshells. Citrate gold nanoparticles had approximately 30\% more penetration at the tumor periphery compared to the gold nanoshells, but this was mostly due to the larger size of the nanoshells, which are three times the size of the citrate gold particles.

\section{Discussion}

We developed two and three layer nanoshells to compare the penetration and diffusion capabilities of these particles against PEGylated AuroShells ${ }^{\mathrm{TM}}$ in $3 \mathrm{D}$ cell cultures. ${ }^{49}$ To this end we used PC, which has previously been employed as a passivating agent for gold nanorods significantly reducing their cytotoxicity. ${ }^{50}$ In addition, $\mathrm{PC}$ is the most abundant phospholipid within the body and can be found in all cell membranes, and is thus expected to be less immunogenic. 

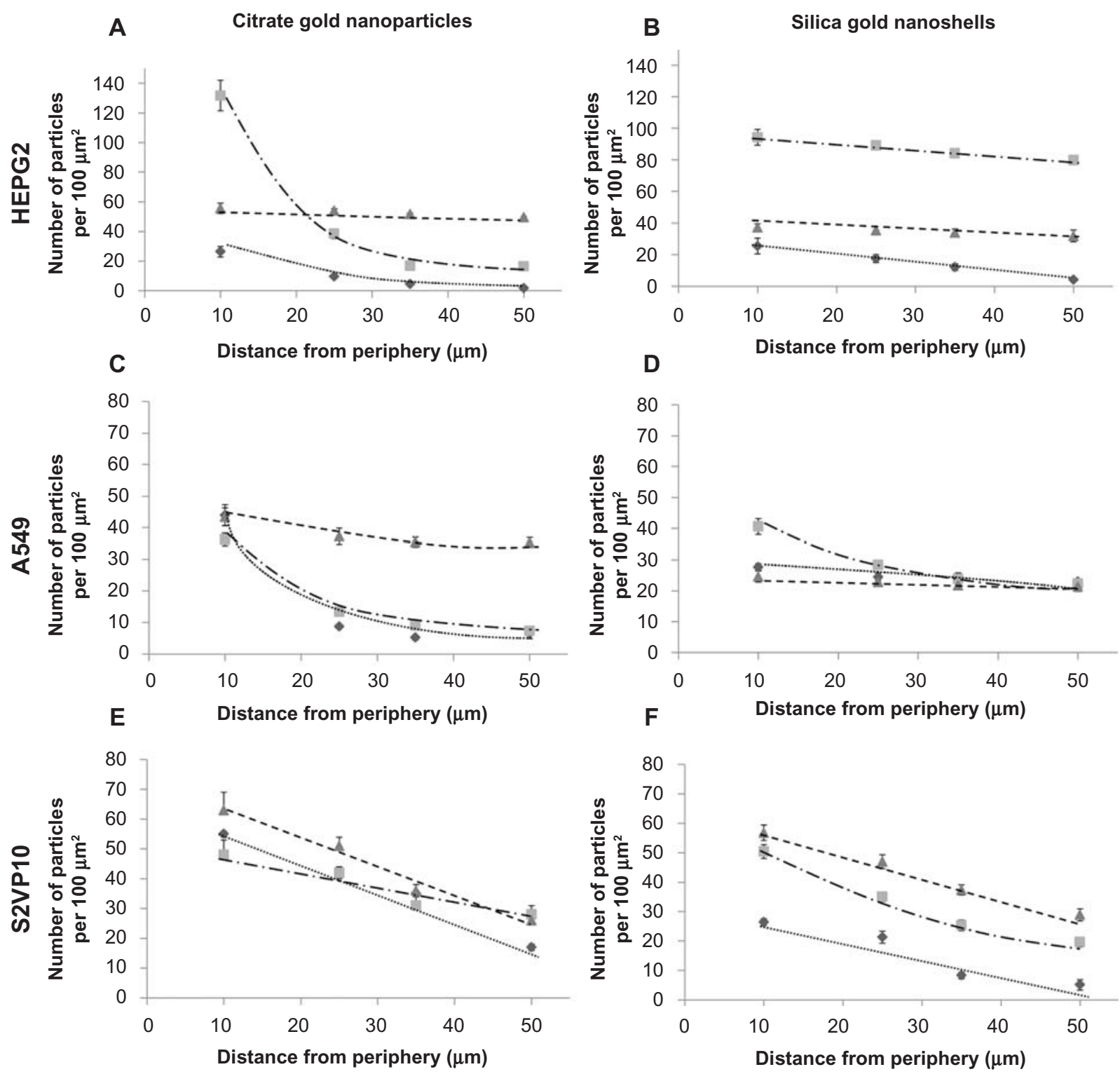

Figure 6 Nanoparticle penetration and distribution in 3D cell cultures as measured by dark field microscopy.

Notes: Diamonds and dotted line: PEGylated; Squares and dashed/dotted line: two layer; Triangles and dashed line: three layer. Particle uptake was quantified for citrate gold nanoparticles (left) and silica gold nanoshells (right) for HEPG2 (liver), A549 (non small cell lung cancer), and S2VPI0 (pancreatic) 3D cell cultures as a function of distance from the spheroid periphery. The two layer silica gold nanoshells in the HEPG2 tumors and the three layer citrate gold nanoparticles in the A549 tumors had the best performance in terms of higher uptake and uniform distribution, while both of these particle types behaved similarly in the S2VPI0 tissue with lower uptake and steeper diffusion gradients. Generally, the PEGylated particles exhibited lower uptake and poorer penetration. All error bars represent standard deviation from at least $\mathrm{n}=3$ measurements.

Abbreviations: 3D, three dimensional; PEG, poly(ethylene)glycol.

In order to form a layer of PC around the gold nanoshells, a hydrophobic layer must also be created. Thiol groups exhibit strong binding to gold, and can be used to displace other stabilizing ligands and adsorbed molecules to stabilize the particles. An alkanethiol possesses optimal qualities for this purpose; the hydrophobic carbon chains will interact with the fatty acids of the PC to form a properly oriented layer. Here, we theorized that such a two layer approach would yield better biocompatibility and thus improved penetration into under-vascularized tumor regions when compared to PEGylated particles. With the two layer method, a hydrophobic region is created between the PC and alkanethiol, which could potentially hold hydrophobic drugs to produce a more potent therapeutic agent. Further, NIRabsorbing gold nanoshells have proven effective at reducing cell viability significantly in solid tumors due to hyperthermia when treated with laser radiation. ${ }^{38,51}$

We find that modifying gold nanoshells with an alkanethiol and phosphatidylcholine creates a viable two layer nanoparticle platform that consistently outperforms the 
current standard, PEGylated nanoparticles, when measuring particle accumulation in 3D cell cultures. As such, two layer nanoshells should be further considered for potential photothermal and targeted drug delivery therapies. The addition of the phosphatidylcholine layer makes the nanoparticles similar to liposomes, which have been previously used for enhanced delivery of gold nanoparticles. ${ }^{52}$ We hypothesize that the phospholipid similarities increased cellular uptake of these nanoparticles as compared to PEGylated nanoparticles. We also developed a three layer system; experimental evidence suggests that HDL uptake in hepatocytes is upregulated in liver cancers. ${ }^{53}$ Adding a layer of HDL around the two layer particles could potentially allow the particles to penetrate deeper into liver cancers as the surface modifications make uptake by the hepatocytes more likely rather than just relying on the EPR effect for passive targeting. The enhanced uptake of HDL in pancreatic and liver cancer suggests increased cellular uptake of HDL-coated nanoparticles. ${ }^{54,55}$ Surface charge has a large effect on how nanoparticles will react in vivo. PEGylated nanoparticles were expected to exhibit slightly negative values similar to those found in literature. Two layer nanoparticles with phosphatidylcholine were expected to exhibit highly negative zeta potentials from $-15-40 \mathrm{mV}$ due to the negative choline head group, while the neutral surface charge of HDL would yield particles with neutral surface charges. ${ }^{56}$ These expectations were confirmed as shown in Figure 2.

FTIR confirmed the identity of the nanoparticles by revealing the presence of various bands. For HDL, bands associated with lipid esters $\left(\sim 1700-1800 \mathrm{~cm}^{-1}\right)$ and two distinct amide stretches $\left(\sim 1500-1700 \mathrm{~cm}^{-1}\right)$ were expected along with characteristics from the FTIR of phosphatidylcholine (due to the layering process of nanoparticles). ${ }^{57} \mathrm{PC}$ coated nanoparticles were expected to have a large peak associated with $-\mathrm{CH}_{2}$ and $-\mathrm{CH}_{3}$ groups $\left(\sim 3000 \mathrm{~cm}^{-1}\right)$ along with phosphate group vibrations $\sim 900 \mathrm{~cm}^{-1} .^{58,59}$ PEGylated nanoparticles were expected to exhibit decreased $-\mathrm{CH}_{2}$, $-\mathrm{CH}_{3}$, and $-\mathrm{CH}$ out of plane bending intensities. These particles were also expected to exhibit a PEG ether stretch C-O-C $\sim 1050-1100 \mathrm{~cm}^{-1}$ with the corresponding vibration band $\sim 1342-1353 \mathrm{~cm}^{-1}{ }^{42,60}$ The bands of the two and three layer nanoparticles were stronger than the PEGylated nanoparticles.

The penetration and diffusion capabilities of two layer and three layer citrate-gold nanoparticles into 3D cell cultures was also analyzed, although the application of these particles in cancer therapy is limited compared to silica-core gold nanoshells. Citrate gold nanoparticles are much smaller than nanoshells and do not possess tunable optical properties; they also lack absorption in the NIR range and thus cannot be used for photothermal ablation therapy.

This study focused on evaluating the uptake and distribution of nanoparticles within avascular/hypoxic regions of solid tumors at varying depths of penetration using 3D cell cultures as an in vitro model of avascular tumor tissue. By quantifying the amount of particles within specific regions of tissue, the diffusion of particles can be analyzed and this information combined with mathematical modeling to better understand effectiveness of these particles for cancer treatment. The results could then be utilized to calibrate computational simulations to elucidate the complex dynamics of particles and drug molecules within solid tumors..$^{11,14,15,61}$ The work here represents an initial step to assess the performance of two and three layered nanoparticles in penetrating avascular tissue. We note that $3 \mathrm{D}$ cell culture is a rudimentary model of avascular tumor tissue; also, the reticuloendothelial system (RES) and other systemic factors may significantly affect nanoparticle performance in vivo.

This study can be continued by examining the efficacy of the two layer nanoshell system in photothermal applications and determining its benefits compared to PEGylated nanoshells. In addition, work is currently in progress to investigate the potential for embedding drugs into the layers of the two layer nanoshell for chemotherapeutic delivery. A more interesting approach would be to combine the photothermal treatments with drug delivery. A system could potentially be designed that would release its drug payload once the particle has been excited through NIR energy, disrupting the layers on the nanoshell. These steps would provide a logical progression towards developing more efficacious systems for targeted cancer therapy.

\section{Acknowledgments}

We acknowledge the IPIBS fellowship at the University of Louisville with support from the Department of Pharmacology and Toxicology and the Department of Bioengineering.

\section{Disclosure}

The authors report no conflicts of interest in this work.

\section{References}

1. Kong G, Braun RD, Dewhirst MW. Hyperthermia enables tumor-specific nanoparticle delivery: effect of particle size. Cancer Res. 2000;60(16): 4440-4445.

2. Maeda $\mathrm{H}$. The enhanced permeability and retention (EPR) effect in tumor vasculature: the key role of tumor-selective macromolecular drug targeting. Adv Enzyme Regul. 2001;41:189-207. 
3. Murphy CJ, Gole AM, Stone JW, et al. Gold nanoparticles in biology: beyond toxicity to cellular imaging. Acc Chem Res. 2008;41(12): 1721-1730.

4. Zamboni WC, Torchilin V, Patri AK, et al. Best practices in cancer nanotechnology: perspective from NCI nanotechnology alliance. Clin Cancer Res. 2012;18(12):3229-3241.

5. Alkilany AM, Murphy CJ. Toxicity and cellular uptake of gold nanoparticles: what we have learned so far? J Nanopart Res. 2010;12(7): 2313-2333.

6. Kennedy LC, Bickford LR, Lewinski NA, et al. A new era for cancer treatment: gold-nanoparticle-mediated thermal therapies. Small. 2011;7(2):169-183.

7. Goodman CM, McCusker CD, Yilmaz T, Rotello VM. Toxicity of gold nanoparticles functionalized with cationic and anionic side chains. Bioconjug Chem. 2004;15(4):897-900.

8. Chabner BA. Cancer Chemotherapy and Biotherapy: Principles and Practice. 5th ed. Philadelphia: Lippincott Williams and Wilkins; 2011.

9. Vinci M, Gowan S, Boxall F, et al. Advances in establishment and analysis of three-dimensional tumor spheroid-based functional assays for target validation and drug evaluation. BMC Biol. 2012;10:29.

10. Krock BL, Skuli N, Simon MC. Hypoxia-induced angiogenesis: good and evil. Genes Cancer. 2011;2(12):1117-1133.

11. van de Ven AL, Wu M, Lowengrub J, et al. Integrated intravital microscopy and mathematical modeling to optimize nanotherapeutics delivery to tumors. AIP Adv. 2012;2(1):11208.

12. Brigger I, Dubernet C, Couvreur P. Nanoparticles in cancer therapy and diagnosis. Adv Drug Deliv Rev. 2002;54(5):631-651.

13. Jain RK. Transport of molecules in the tumor interstitium: a review. Cancer Res. 1987;47(12):3039-3051.

14. van de Ven A, Abdollahi B, Martinez C, et al. Modeling of nanotherapeutics delivery based on tumor perfusion. New J Phys. 2013;15: 055004.

15. Wu M, Frieboes HB, McDougall SR, Chaplain MA, Cristini V, Lowengrub J. The effect of interstitial pressure on tumor growth: coupling with the blood and lymphatic vascular systems. J Theor Biol. 2013;320:131-151.

16. Michor F, Liphardt J, Ferrari M, Widom J. What does physics have to do with cancer? Nat Rev Cancer. 2011;11(9):657-670.

17. Wong C, Stylianopoulos T, Cui J, et al. Multistage nanoparticle delivery system for deep penetration into tumor tissue. Proc Natl Acad Sci USA. 2011;108(6):2426-2431.

18. Goodman TT, Olive PL, Pun SH. Increased nanoparticle penetration in collagenase-treated multicellular spheroids. Int $J$ Nanomedicine. 2007;2(2):265-274.

19. Huang K, Ma H, Liu J, et al. Size-dependent localization and penetration of ultrasmall gold nanoparticles in cancer cells, multicellular spheroids, and tumors in vivo. ACS Nano. 2012;6(5):4483-4493.

20. Cabral H, Matsumoto Y, Mizuno K, et al. Accumulation of sub-100 nm polymeric micelles in poorly permeable tumours depends on size. Nat Nanotechnol. 2011;6(12):815-823.

21. Garrett N, Whiteman M, Moger J. Imaging the uptake of gold nanoshells in live cells using plasmon resonance enhanced four wave mixing microscopy. Opt Express. 2011;19(18):17563-17574.

22. Liang Z, Liu Y, Li X, et al. Surface-modified gold nanoshells for enhanced cellular uptake. J Biomed Mater Res A. 2011;98(4):479-487.

23. Chithrani BD, Ghazani AA, Chan WC. Determining the size and shape dependence of gold nanoparticle uptake into mammalian cells. Nano Lett. 2006;6(4):662-668.

24. Frieboes HB, Edgerton ME, Fruehauf JP, et al. Prediction of drug response in breast cancer using integrative experimental/computational modeling. Cancer Res. 2009;69(10):4484-4492.

25. Srinivas A, Rao PJ, Selvam G, Murthy PB, Reddy PN. Acute inhalation toxicity of cerium oxide nanoparticles in rats. Toxicol Lett. 2011;205(2): 105-115.

26. Frens G. Controlled nucleation for the regulation of the particle size in monodisperse Gold solutions. Nature Phys Sci. 1973;241:20-22.
27. Stöber W, Fink A, Bohn E. Controlled growth of monodisperse silica spheres in the micron size range. J Colloid Interface Sci. 1968;26(1): $62-69$.

28. Kalele S, Gosavi SW, Urban J, Kulkarni AK. Nanoshell particles: synthesis, properties and applications. Current Sci. 2006;91(8):1038-1052.

29. Preston TC, Signorell R. Growth and optical properties of gold nanoshells prior to the formation of a continuous metallic layer. ACS Nano. 2009;3(11):3696-3706.

30. Duff DG, Baiker A, Edwards PP. A new hydrosol of gold clusters. 1. Formation and particle size variation. Langmuir. 1993;9(9):2301-2309.

31. Guan M, Zhou Y, Zhu QL, et al. N-trimethyl chitosan nanoparticleencapsulated lactosyl-norcantharidin for liver cancer therapy with high targeting efficacy. Nanomedicine. 2012;8(7):1172-1181.

32. Wang J, Zhang Z, Wang X, Wu W, Jiang X. Size- and pathotropismdriven targeting and washout-resistant effects of boronic acid-rich protein nanoparticles for liver cancer regression. J Control Release. 2013;168(1):1-9.

33. Lai LF, Guo HX. Preparation of new 5-fluorouracil-loaded zein nanoparticles for liver targeting. Int J Pharm. 2011;404(1-2):317-323.

34. Kimling J, Maier M, Okenve B, Kotaidis V, Ballot H, Plech A. Turkevich method for gold nanoparticle synthesis revisited. J Phys Chem B. 2006;110(32):15700-15707.

35. Leung K. Gold-polyethylene glycol nanoshells. In: Molecular Imaging and Contrast Agent Database (MICAD) [Internet]. Bethesda: National Center for Biotechnology Information (US); 2004-2013.

36. Tullman JA, Finney WF, Lin YJ, Bishnoi SW. Tunable assembly of peptide-coated gold nanoparticles. Plasmonics. 2007;2(3):119-127.

37. O'Neal DP, Hirsch LR, Halas NJ, Payne JD, West JL. Photo-thermal tumor ablation in mice using near infrared-absorbing nanoparticles. Cancer Lett. 2004;209(2):171-176.

38. Gobin AM, Lee MH, Halas NJ, James WD, Drezek RA, West JL. Near-infrared resonant nanoshells for combined optical imaging and photothermal cancer therapy. Nano Lett. 2007;7(7):1929-1934.

39. Jokerst JV, Lobovkina T, Zare RN, Gambhir SS. Nanoparticle PEGylation for imaging and therapy. Nanomedicine (Lond). 2011;6(4): 715-728.

40. Maldiney T, Richard C, Seguin J, Wattier N, Bessodes M, Scherman D. Effect of core diameter, surface coating, and PEG chain length on the biodistribution of persistent luminescence nanoparticles in mice. ACS Nano. 2011;5(2):854-862.

41. Gu X, Zhang W, Liu J, et al. Preparation and characterization of a lovastatin-loaded protein-free nanostructured lipid carrier resembling high-density lipoprotein and evaluation of its targeting to foam cells. AAPS Pharm Sci Tech. 2011;12(4):1200-1208.

42. Wen SH, Zheng F, Shen M, Shi X. Surface modification and PEGylation of branched polyethyleneimine for improved biocompatibility. $\mathrm{J} \mathrm{Appl}$ Polymer Sci. 2013;128(6):3807-3813.

43. Lewis BA, Engelman DM. Lipid bilayer thickness varies linearly with acyl chain length in fluid phosphatidylcholine vesicles. $J$ Mol Biol. 1983;166(2):211-217.

44. Nagle JF, Tristram-Nagle S. Structure of lipid bilayers. Biochim Biophys Acta. 2000;1469:159-195.

45. El Harchaoui K, Arsenault BJ, Franssen R, et al. High-density lipoprotein particle size and concentration and coronary risk. Ann Intern Med. 2009;150(2):84-93.

46. Kumar C, editor. Mixed Metal Nanomaterials. Weinheim: Wiley-VCH Verlag GmbH; 2009.

47. Rothe DR, Wood SP, Heeschen WA. Imaging of submicron particulate in an optical flow cell. Microsc Microanal. 2007;13(Suppl S02):672-673.

48. Yen JC, Chang FJ, Chang S. A new criterion for automatic multilevel thresholding. IEEE Trans Image Process. 1995;4(3):370-378.

49. Nanospectra Nanosciences, Inc [homepage on the Internet]. Available from: http://www.nanospectra.com. Accessed April 15, 2013.

50. Takahashi H, Niidome Y, Niidome T, Kaneko K, Kawasaki H, Yamada S. Modification of gold nanorods using phosphatidylcholine to reduce cytotoxicity. Langmuir. 2006;22(1):2-5. 
51. Lowery AR, Gobin AM, Day ES, Halas NJ, West JL. Immunonanoshells for targeted photothermal ablation of tumor cells. Int J Nanomedicine. 2006;1(2):149-154.

52. Kojima C, Hirano Y, Kono K. Chapter 7 - Preparation of complexes of liposomes with gold nanoparticles. Meth Enzymol. 2009;464: 131-145.

53. Jiang J, Nilsson-Ehle $\mathrm{P}, \mathrm{Xu} \mathrm{N}$. Influence of liver cancer on lipid and lipoprotein metabolism. Lipids Health Dis. 2006;5:4.

54. Shahzad MM, Mangala LS, Han HD, et al. Targeted delivery of small interfering RNA using reconstituted high-density lipoprotein nanoparticles. Neoplasia. 2011;13(4):309-319.

55. Ng KK, Lovell JF, Zheng G. Lipoprotein-inspired nanoparticles for cancer theranostics. Acc Chem Res. 2011;44(10):1105-1113.

56. Blume G, Cevc G. Molecular mechanism of the lipid vesicle longevity in vivo. Biochim Biophys Acta. 1993;1146(2):157-168.
57. Krilov D, Balarin M, Kosović M, Gamulin O, Brnjas-Kraljević J. FT-IR spectroscopy of lipoproteins - a comparative study. Spectrochim Acta A Mol Biomol Spectrosc. 2009;73(4):701-706.

58. Semalty A, Semalty M, Singh D, Rawat MSM. Preparation and characterization of phospholipid complexes of naringenin for effective drug delivery. J Incl Phenom Macrocycl Chem. 2010;67:253-260.

59. Wang F, Yang ZL, Zhang L, Weng SF, Wu JG. FT-IR study of the interaction between phosphatidylcholine and bovine serum albumin. Acta Physico-Chimica Sinica. 2004;20:1186-1190.

60. Gupta AK, Curtis AS. Surface modified superparamagnetic nanoparticles for drug delivery: interaction studies with human fibroblasts in culture. J Mater Sci Mater Med. 2004;15(4):493-496.

61. Frieboes HB, Wu M, Lowengrub J, Decuzzi P, Cristini V. A computational model for predicting nanoparticle accumulation in tumor vasculature. PLoS One. 2013;8(2):e56876. 


\section{Supplementary materials}
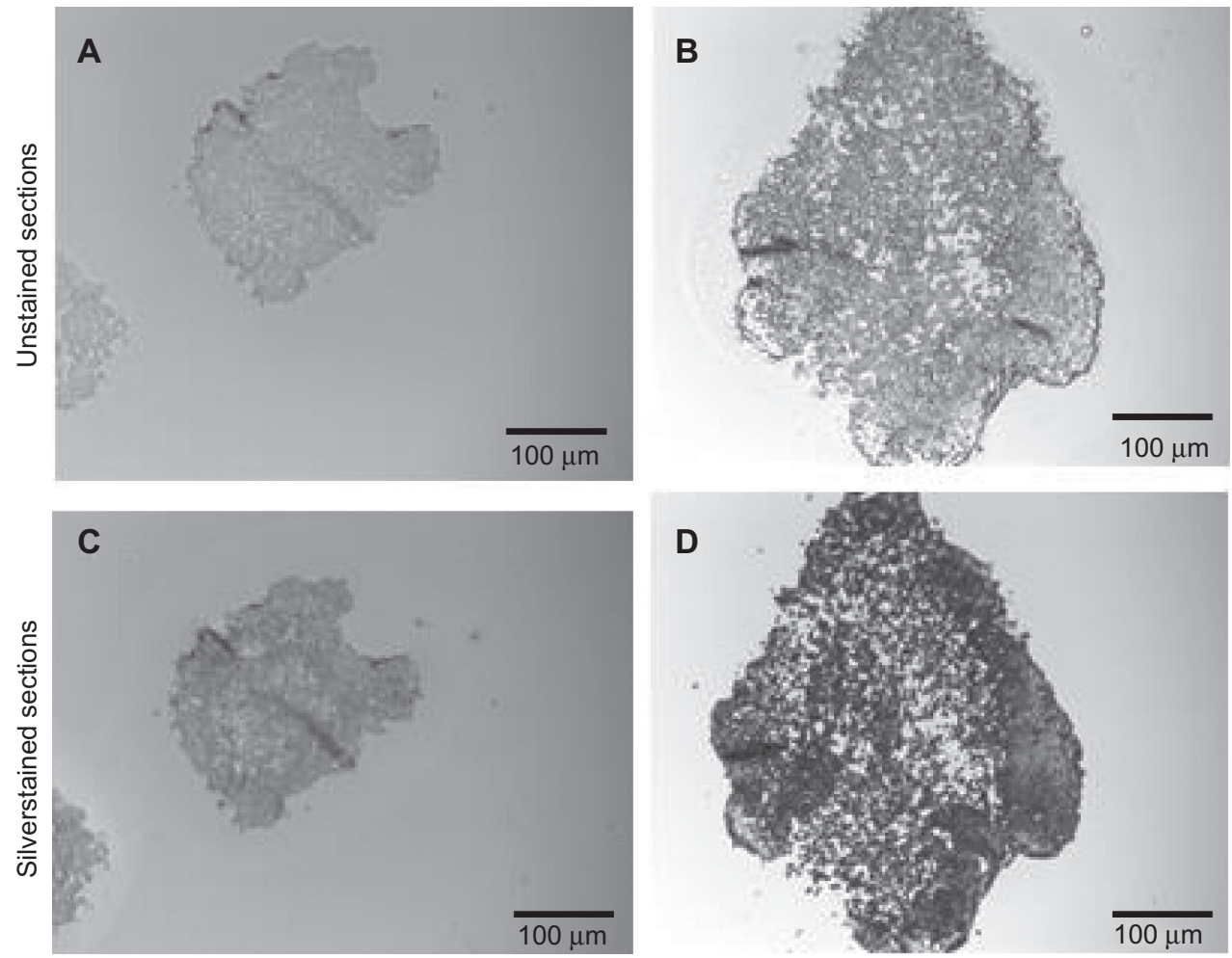

Figure SI Nanoparticle detection in 3D cell cultures through silver staining.

Notes: ROI intensities were measured for both unstained and stained tissue sections; darker regions denote an increase in ROI intensity. (A) $20 \times$ image of $A 5493 D$ cell culture before and (B) after silver staining. (C) 20x image of HEPG2 3D cell culture before and (D) after silver staining.

Abbreviations: 3D, three dimensional; ROI, region of interest.
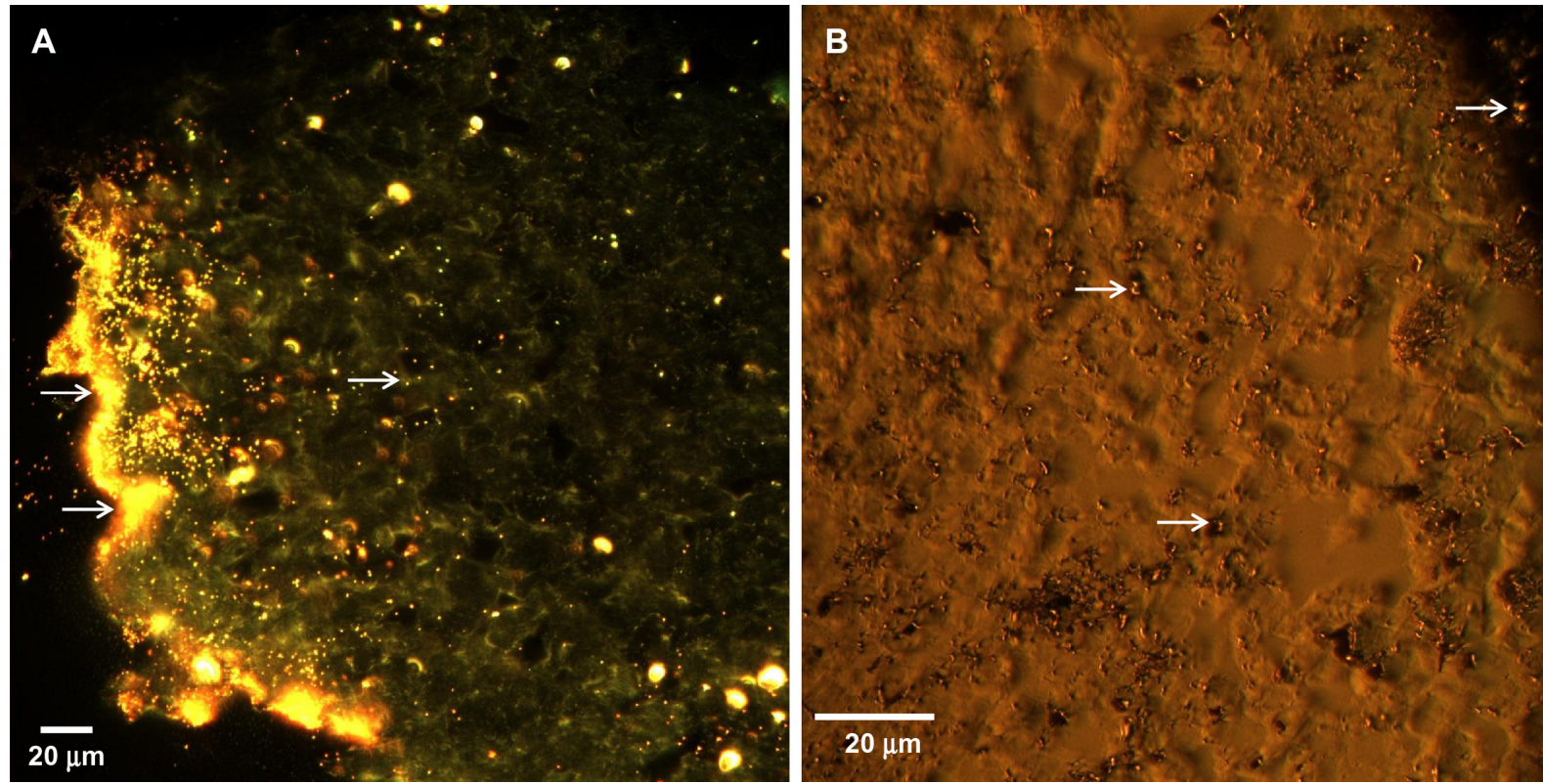

Figure S2 Detection of nanoparticles within 3D cell cultures using dark field microscopy.

Notes: Nanoparticle uptake was determined using the CytoViva setup with a dark field microscope to visualize the nanoparticles, and with hyperspectral imaging to confirm particle identity. (A) 60X image showing increased particle concentration around periphery of HEPG2 3D cell culture with decreasing particle numbers towards the interior. (B) I00× image of A549 3D cell culture showing the particles (arrows) distributed throughout the tissue.

Abbreviation: 3D, three dimensional. 

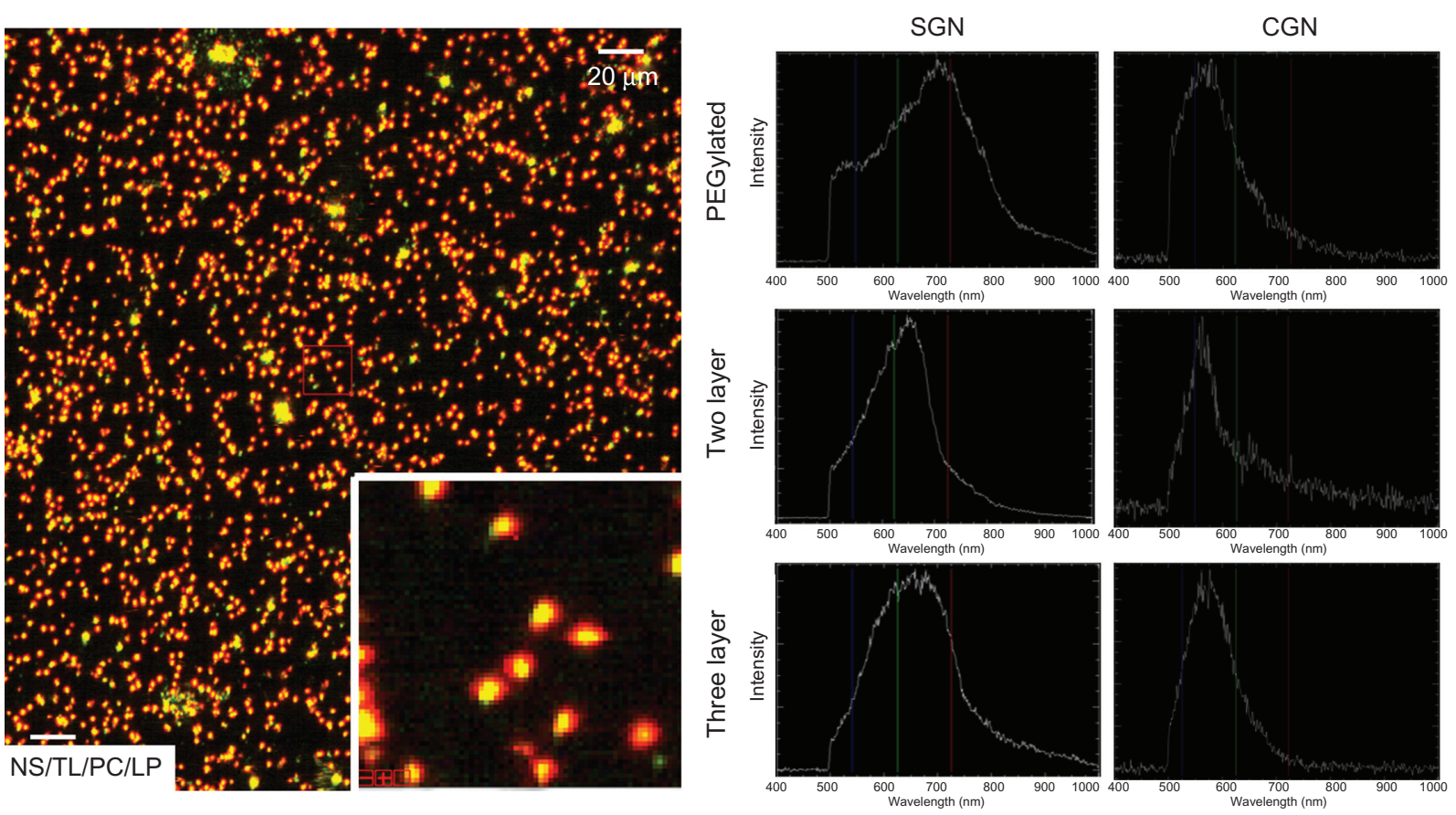

Figure S3 Hyperspectral imaging of nanoparticles using CytoViva hyperspectral imaging system.

Notes: Nanoparticles in solution were placed on slide for analysis using hyperspectral imaging. Individual particles could be identified using this technique. For further analysis of the particles, individual spectra are presented for the PEGylated, and two and three layer nanoparticles. Spectra differences are associated with varying surface modifications.

Abbreviations: CGN, Citrate Gold Nanoparticle; NS, nanoshell; PC, phosphatidylcholine; PEG, poly(ethylene)glycol; SGN, Silica Gold Nanoshell; TL, thiol; LP, lipoprotein.

\section{Publish your work in this journal}

The International Journal of Nanomedicine is an international, peerreviewed journal focusing on the application of nanotechnology in diagnostics, therapeutics, and drug delivery systems throughou the biomedical field. This journal is indexed on PubMed Central, MedLine, CAS, SciSearch ${ }^{\circledR}$, Current Contents ${ }^{\circledR} /$ Clinical Medicine,
Journal Citation Reports/Science Edition, EMBase, Scopus and the Elsevier Bibliographic databases. The manuscript management system is completely online and includes a very quick and fair peer-review system, which is all easy to use. Visit http://www.dovepress.com/ testimonials.php to read real quotes from published authors. 